\title{
Solving binary classification problems by means of convolutional neural networks with the use of TensorFlow framework
}

Oleksiy Oleksandrovych Denysenko, software developer

individual entrepreneur Oleksiy Oleksandrovych Denysenko (Kyiv, Ukraine)

This article is devoted to the task of classifying cats and dogs using aconvolutional neural network and Tensorflow framework.

Keywords: convolutional neural network, Tensorflow, classification, machine learning.

Classification is an integral part of everyone's life. Day after day, the human brain classifies many things with incredible accuracy andease.
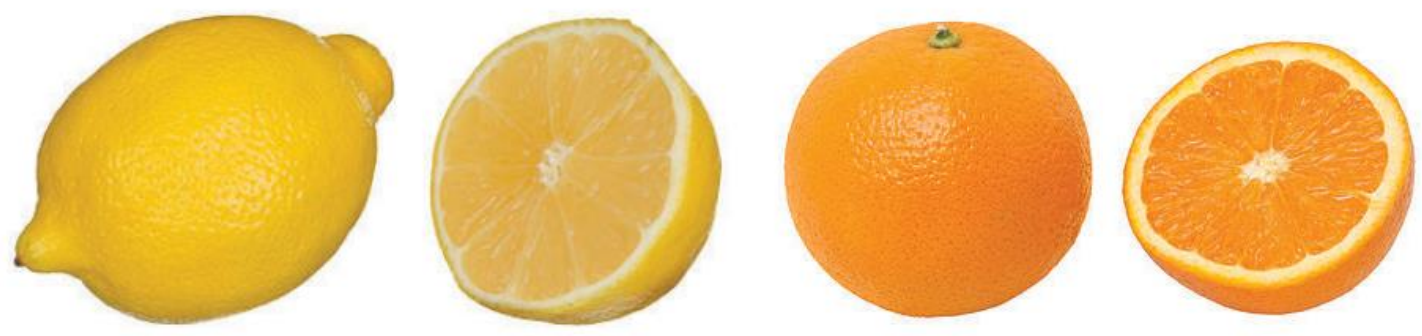

Fig. 1. A lemon and an orange

If you use basic conditional operators, you may need Buta trained neural network can easily perform such a a thousand conditions to distinguish a lemon from an task. Classification of irises is one of the popular orange. It is necessary to take into account the color and classification tasks [1]. size, shape, pattern on the peel, etc.

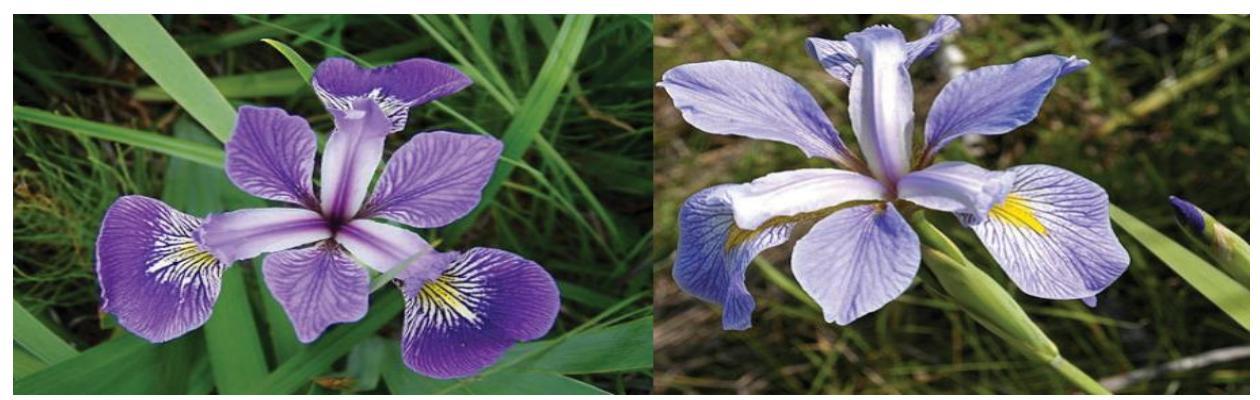

Fig. 2. Iris Versicolor and Iris Virginica 
You can classify irises based on just four unique parameters (length and width of the sepal, length and width of the petal). The task of image classification is solved with the help of convolutional neural networks.

The convolutional neural network, which was proposed by Yann LeCun in 1988, is a special architecture of artificial neural networks, which is designed for effective image recognition and is part of the deep learning technologies [2]. The network architecture got its name dueto the use of the convolution operation, which is based on the fact that every fragment of an image is multiplied by the convolution matrix (kernel) element by element, and the result is summed up and returned to the corresponding position in the output image.

Let us consider how one can solve the problem of classifying cats and dogs with the help of the convolutional neural network. The tensorflow framework will be used when building the network.

The initial data set should be prepared before processing, all images must be grayscale and must not exceed the size of $70 \times 70$ pixels. This will help accelerate the process of training the neural network, and we will not use excessive resources of the operating system.

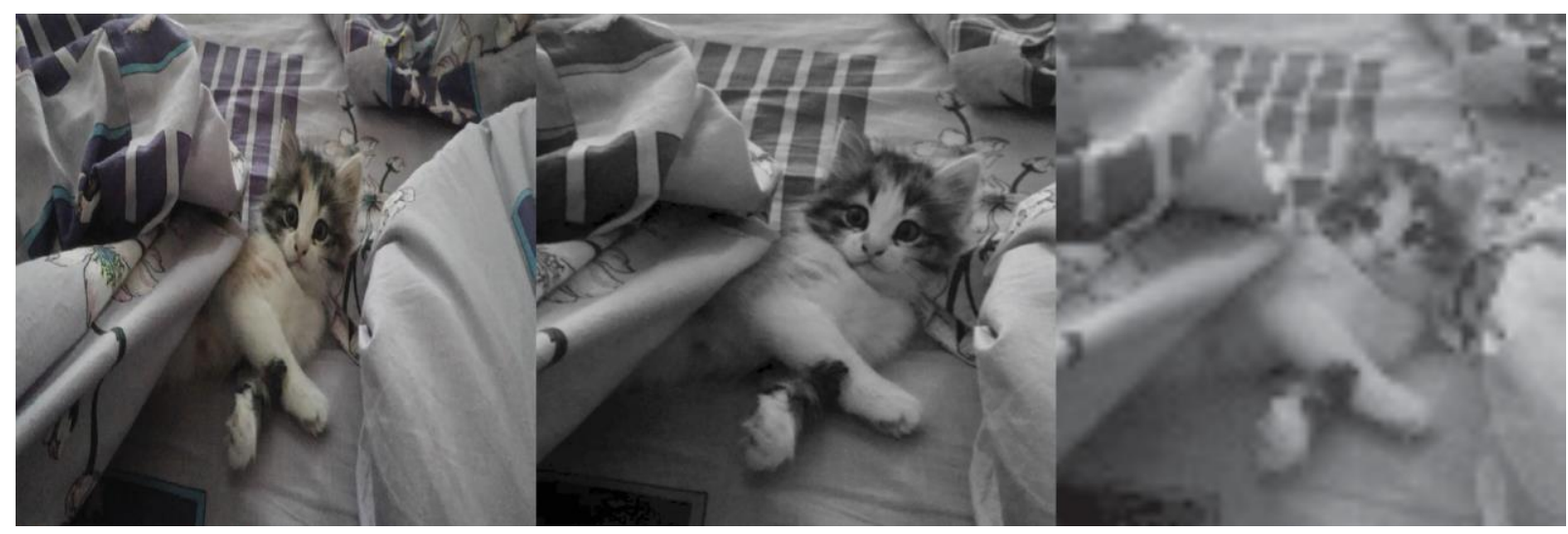

Fig . 3. Stages of image preprocessing (from left to right: original image, grayscale image, image the size of which has been reduced to $70 \times 70$ pixels)

Network topology. Since this task belongs to the 1,2, or 3 convolutional layers, each of which consisted of category of binary classification - the task of classifying 32,64 , or 128 neurons, for each of which 0,1 , or 2 various elements into two groups - we have only two subsampling layers were used. The experiments showed input neurons.

A hidden layer was chosen by experiment.

The following combinations were tested in the course of the experiment: that networks with three convolutional layers for which a subsampling layer was not used produced the best results (Fig. 4).

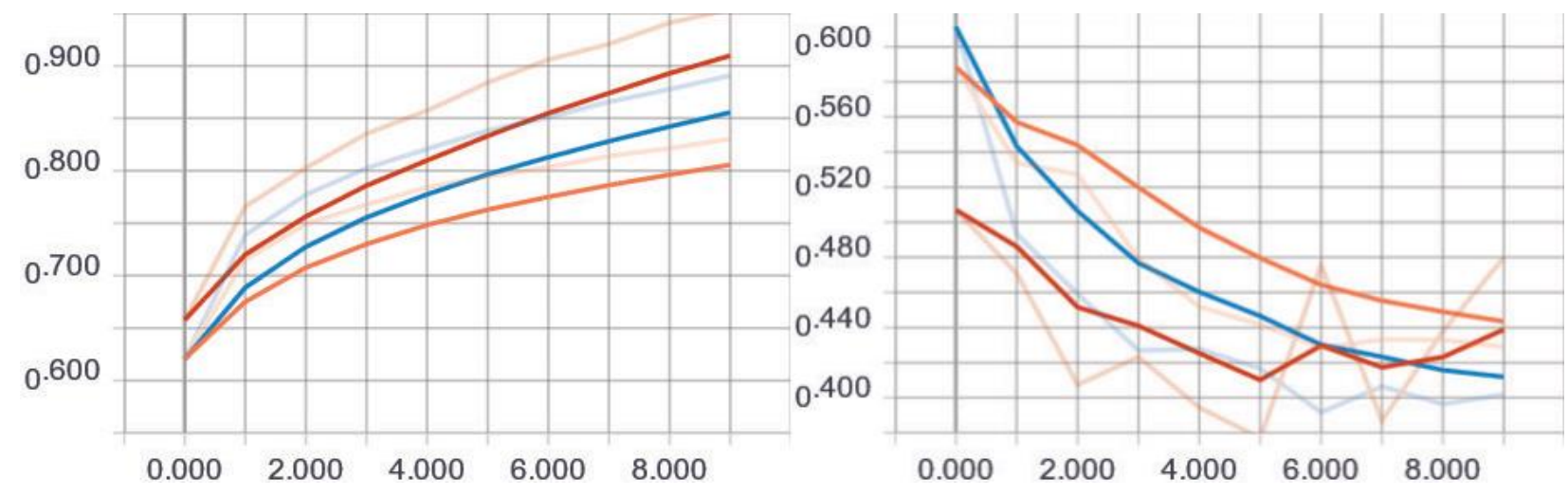

Fig . 4. Prediction accuracy (on the left) and network error rate (on the right)

Despite the fact that the network with three convolutional layers and 128 neurons (red line) had the best prediction accuracy, a network with three convolutional layers and 64 neurons (blue line) has the lowest error rate.

The scalar result of every convolution corresponds to the activation function, which is a particular non-linear 
function. This can be any function. But hyperbolic calculating the function $f(x)=\max (0, x)$.

tangent functions $f(x)=\tanh x, f(x)=|\tanh x|$, or That is, this is actually the operation of cutting the sigmoid functions $f(x)=\left(1+e^{-x}\right)^{-1}$, were traditionally negative part of the scalar value. As at 2017 , this function used for this task.

and its modifications (Noisy ReLU, Leaky ReLU and

But in the 2000s, a new activation function, ReLU others) are the most frequently used activation functions (rectified linear unit) [3], was proposed and studied, in deep neural networks, in particular, in convolutional which made it possible to significantly speed up the networks. But we will use a usual ReLU function when learning process and simplify calculations (due to the solving this problem. simplicity of the function itself), which means that a

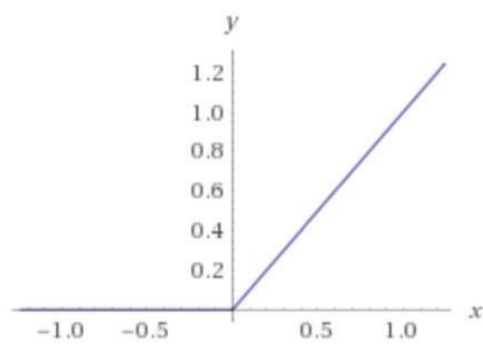

Fig . 5. Plot of the rectified linear unit function

For the output layer, you need to first perform subsampling. The pooling layer, or subsampling layer, is a non-linear compression of a feature map, where a group of pixels (which usually have the size $2 x^{2}$ ) are compressed to one pixel,undergoing non-linear transformation. The main idea of pooling lies in the fact that if some features have already been detected during the previous convolution operation, then such a detailed image is no longer needed for further processing, and it is compressed to a less detailed one.

A sigmoid function was chosen as the activation function for the pooling layer. A sigmoid is a smooth Sshaped monotonically increasing nonlinear function, which is often used for "antialiasing" values of a certain parameter. A logistic function $\sigma(x)=\frac{1}{1+e^{-x}}$

is often meant by a sigmoid function.

For network training, a data set consisting of 22,451 images was used, and validation, in turn, was performed in case of 2,495 images.

The results of this experiment showed that the neural network with three convolutional layers consisting of 64 neurons each, the ReLU activation function, allows one to perform the best classification of cats and dogs based on images. In the course of the experiment, we developed a model of a neural network which can identify with an accuracy of $87 \%$ (Fig. 6) whether a cat or a dog is depicted in an image.

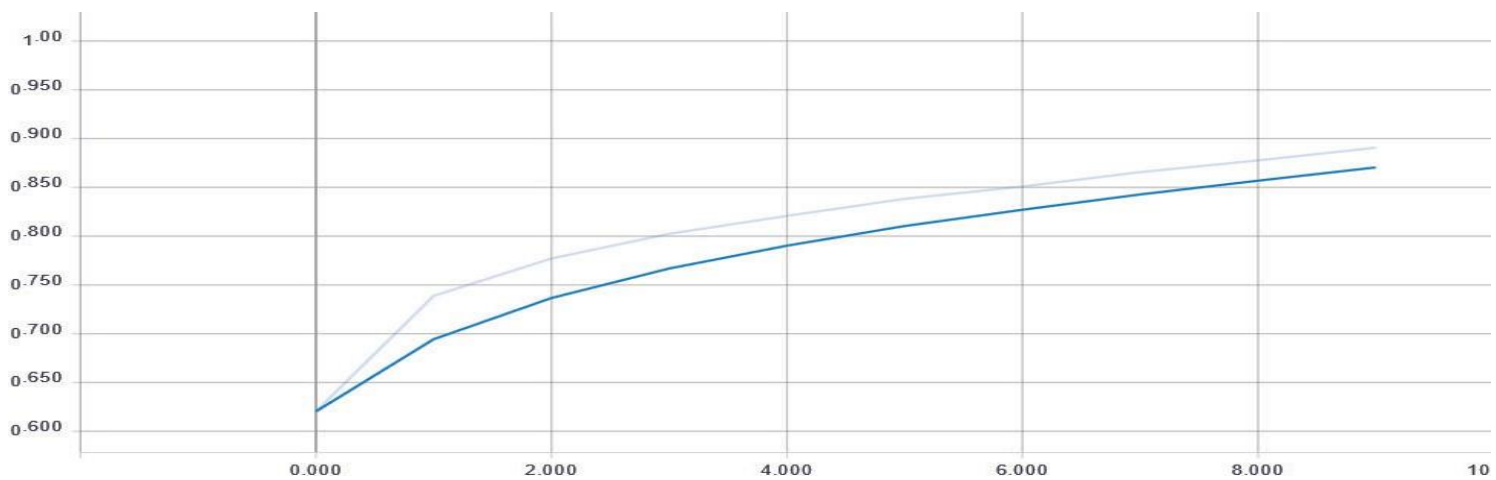

Fig . 6. Accuracy of the resulting network 
References:

1. Fisher, R.A. (1936). "The Use of Multiple Measurements in Taxonomic Problems". Annals of Eugenics. 7: $179-188$.

2. Y. LeCun, B. Boser, J. S. Denker, D. Henderson, R. E. Howard, W. Hubbard and L. D. Jackel: Backpropagation Applied to Handwritten Zip Code Recognition, Neural Computation, 1(4):541-551, Winter 1989.

3. Hahnloser RH, Sarpeshkar R, Mahowald MA, Douglas RJ, Seung HS. 2000. Digital selection and analogue amplification coexist in a cortex-inspired silicon circuit. Nature. 405(6789):947-51. 